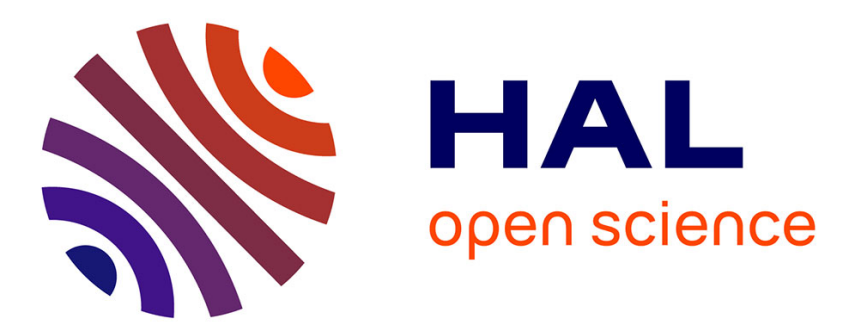

\title{
Jeux numériques pour l'apprentissage: jouer avec la langue ou créer par la langue
}

Joséphine Rémon

\section{To cite this version:}

Joséphine Rémon. Jeux numériques pour l'apprentissage: jouer avec la langue ou créer par la langue. Recherche et pratiques pédagogiques en langues de spécialité - Cahiers de l'APLIUT, 2017, Jeux et langues dans l'enseignement supérieur, 36 (2). hal-01551045

\section{HAL Id: hal-01551045 \\ https://hal.science/hal-01551045}

Submitted on 29 Jun 2017

HAL is a multi-disciplinary open access archive for the deposit and dissemination of scientific research documents, whether they are published or not. The documents may come from teaching and research institutions in France or abroad, or from public or private research centers.
L'archive ouverte pluridisciplinaire HAL, est destinée au dépôt et à la diffusion de documents scientifiques de niveau recherche, publiés ou non, émanant des établissements d'enseignement et de recherche français ou étrangers, des laboratoires publics ou privés. 


\title{
Recherche et pratiques pédagogiques en langues de
} spécialité

Cahiers de l'Apliut

Vol.36 N² | 2017

Jeux et langues dans l'enseignement supérieur

\section{Jeux numériques pour l'apprentissage : jouer avec la langue ou créer par la langue}

Digital games for learning: Playing with language or creating through language

\author{
Joséphine Rémon
}

\section{Q revues.org $\quad \begin{aligned} & \text { Éditeur } \\ & \text { APLIUT }\end{aligned}$}

Édition électronique

URL : http://apliut.revues.org/5675

ISSN : 2119-5242

Référence électronique

Joséphine Rémon, « Jeux numériques pour l'apprentissage : jouer avec la langue ou créer par la

langue », Recherche et pratiques pédagogiques en langues de spécialité [En ligne], Vol.36 №2 | 2017, mis en ligne le 19 juin 2017, consulté le 28 juin 2017. URL : http://apliut.revues.org/5675

Ce document a été généré automatiquement le 28 juin 2017.

Association des Professeurs de Langues des Instituts Universitaires de Technologie 


\title{
Jeux numériques pour \\ l'apprentissage : jouer avec la langue ou créer par la langue
}

Digital games for learning: Playing with language or creating through language

\author{
Joséphine Rémon
}

\section{Introduction}

1 Dans l'optique de redonner toute leur place aux jeux dans l'enseignement/apprentissage, nous présentons dans cette contribution une série de ressources numériques dont l'enseignant peut se saisir pour un travail sur la langue étrangère, plus spécifiquement l'anglais, notamment avec des étudiants spécialistes d'autres disciplines. Nous souhaitons donner des exemples de jeux numériques pouvant être utilisés dans cette pratique de la langue mais aussi fournir quelques outils conceptuels permettant d'appréhender ces ressources. Nous proposons une classification empirique en fonction du rapport d'usager ou de créateur à la ressource et à la langue.

2 Au-delà de la fascination pour l'écran, les ressources numériques présentées ici offrent des avantages d'un point de vue pédagogique. Elles ont en effet en commun de permettre une utilisation transversale, ce qui permet de s'en saisir quelle que soit la discipline, de s'adapter donc à toutes les filières mais aussi à tous les âges. Une autre caractéristique intéressante de ces ressources est qu'elles peuvent être utilisées en salle informatique mais aussi dans des configurations où la salle n'est équipée que d'un poste «maître ». 


\section{Quelques principes opérationnels}

\subsection{Dictée à l'adulte}

3 principe utilisé sera le plus souvent celui de la « dictée à l'adulte » ou de la « délégation d'écriture » (Chartier et al 94), qui devient en quelque sorte une « dictée à l'opérateur du poste informatique », qu'il soit enseignant ou étudiant.

4 Ainsi, typiquement, les jeux de " pointer et cliquer» («point and click»), peuvent être utilisés selon le modèle de la délégation d'action à l'opérateur du poste informatique. Toute fiction interactive graphique ou textuelle peut également être utilisée selon ce mode.

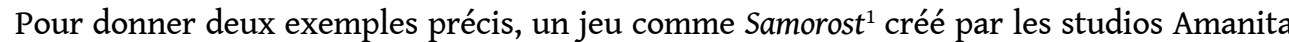
Design (2003), et la suite Botanicula (Amanita Design 2012), peuvent être l'occasion de donner des instructions en langue cible à la personne qui actionne la souris. De même, tout jeu de type « Escape Game » (jeu d'évasion) (par exemple Escaping the Prison ${ }^{2}$, Puffballs United 2010), dans lequel il faut sortir d'un espace clos, peut aussi être l'occasion d'indiquer à la personne maniant la souris des actions qui la feront éventuellement sortir. Ainsi, même si le jeu n'est pas conçu au départ pour faire manier le langage, il peut en devenir l'occasion. Par ailleurs, le principe ludique de "s'échapper d'un lieu en résolvant des énigmes nous semble pouvoir être mis en place par l'enseignant, sans ressources numériques, dans une salle de classe, tout comme il est exploité par des compagnies privées en " grandeur nature ${ }^{3}$, et être l'occasion de manipuler la langue.

Un logiciel comme Lego Builder ${ }^{4}$ (LEGO 2004, figure 1) est aussi typiquement utilisable avec un seul poste informatique sur le principe de la délégation d'action.

Figure 1 : Lego Digital Designer, interface de construction

7 Ce logiciel permet de construire bâtiments ou véhicules et peut être utilisé notamment en lien avec les sciences de l'ingénieur (Erwin et al.).

8 Des jeux-questionnaires (quiz) prêts à l'emploi tels que FreeRice 5 (John Breen 2007), Questionaut $^{6}$ (BBC/Amanita Design 2003), ou encore les quiz de l'encyclopédie Britannica ${ }^{7}$ peuvent être utilisés en autonomie ou en interaction en groupe-classe. Ces jeux offrent l'avantage d'être dans une approche EMILE (Enseignement d'une Matière Intégré à une Langue Etrangère) ou CLIL (Content and Language Integrated Learning), puisqu'ils permettent de travailler les mathématiques en anglais ou des éléments de culture générale en anglais, dans le domaine de l'art par exemple.

\subsection{Combinatoire}

Un autre principe qui donne une meilleure appréhension de ces ressources numériques est celui de la "combinatoire ». En effet, nombreux sont les jeux qui exploitent le principe de l'opposition classique entre syntagme et paradigme telle qu'elle est définie par Saussure: l'axe paradigmatique est le plan vertical des associations, le paradigme désignant l'ensemble des unités entretenant entre elles un rapport virtuel de substitution, tandis que l'axe syntagmatique est le plan horizontal des combinaisons. Exploiter le principe de la combinatoire sur les deux axes permet de proposer des

Recherche et pratiques pédagogiques en langues de spécialité, Vol.36 N² | 2017 
activités pour lesquelles il n'y a pas de réponse prédéterminée, mais un ensemble de possibles. C'est le principe du jeu "cadavre exquis », appliqué dans certains albums de jeunesse, tels que ceux de Sharratt et Robinson en anglais (figure 2).

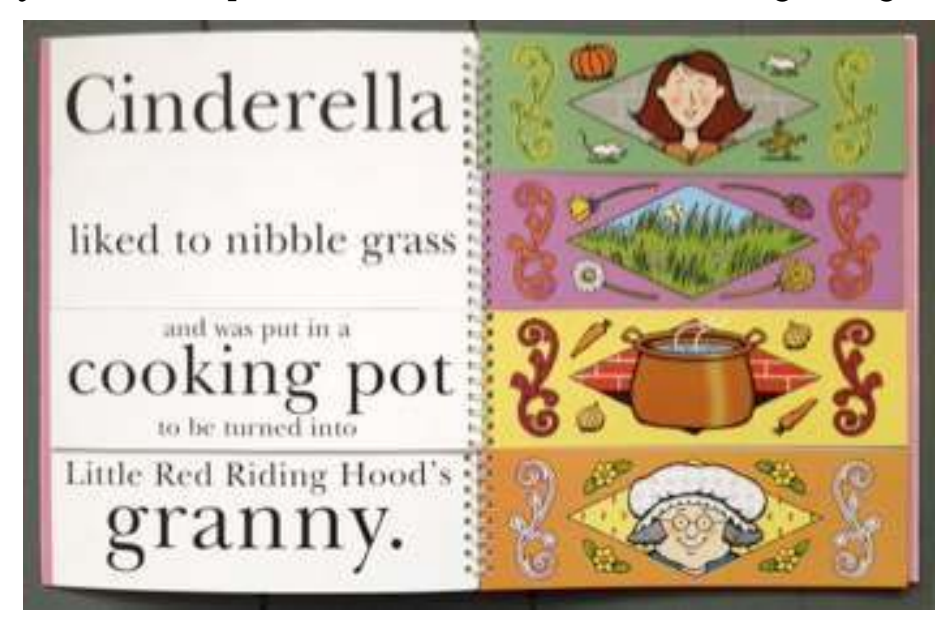

Figure 2 : Sharrat et Robinson (2005), Mixed-up fairy tales

10 Ce principe est très puissant pour les ressources numériques à potentiel éducatif. Ainsi, Super Hero $\mathrm{Me}^{8}$ (DC Comics 2017), utilisé en collège notamment, permet aux élèves de créer un super héro, mais d'autres générateurs tels que MakeBeliefsComix ${ }^{9}$ (Bill Zimmerman, Guarionex Press Ldt 2006-2017) pour la création d'une bande dessinée ou Fodey ${ }^{10}$ (Christian Hirche 2006), pour la création d'une Une de journal, d'un paquet de cigarettes ou d'un écureuil parlant, s'adaptent à tous les publics.

\section{Jouer par le langage}

Une autre catégorie de ressources numériques ludiques permet non seulement de pratiquer la langue, mais ne fonctionne qu'à travers la langue. Ainsi, l'apprenant n'a d'autre choix que de produire du langage pour participer. La tâche comprend en ellemême une contrainte créative. On pourrait y voir un autre cas de "délégation d'action ", avec cette fois une action verbale.

\subsection{Fiction interactive}

La fiction interactive est une histoire, une aventure, un scénario qui ne progresse que par l'intervention de l'utilisateur. Dans certains cas, il ne s'agit que de cliquer sur une des options proposées, la compétence mobilisée étant alors la compréhension écrite (par exemple, ci-dessous, figure 3, Arsonsaurus ${ }^{11}$ de Mike Meyer 2015).

Figure 3 : Arsonsaurus, (Mike Meyer 2015) : fiction interactive par liens hypertextes, interface de jeu.

Dans The Hitchhiker's Guide to the Galaxy ${ }^{12}$ (1984), créé par la $B B C$, d'après le film culte du même nom, l'utilisateur est plongé dans le noir, et doit, par des instructions écrites au clavier, petit à petit se lever, allumer la lumière, et au plus vite comprendre quel danger le menace (figure 4). 


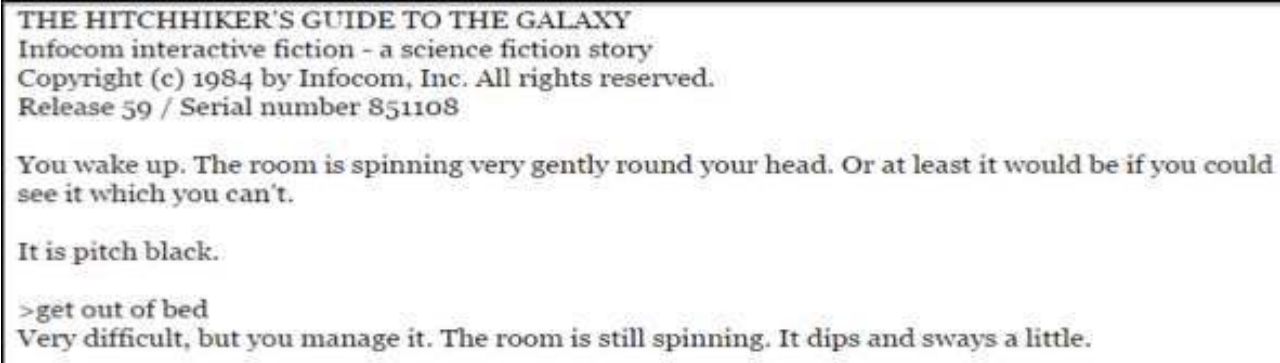

Figure 4 : The Hitchhiker's Guide to the Galaxy (BBC 1984), introduction dans l'interface de jeu.

De même, dans le jeu Her Story ${ }^{13}$ (Sam Barlow 2015, figure 5), qui peut être acheté pour une somme modique par les étudiants, l'enseignant ou l'institution, le joueur ne peut progresser dans la résolution d'une enquête policière que par l'intermédiaire de mots clés, qu'il utilise pour interroger une base de données de courtes vidéos. Chaque vidéo ainsi récupérée donne des indices pour rebondir vers d'autres mots clés.

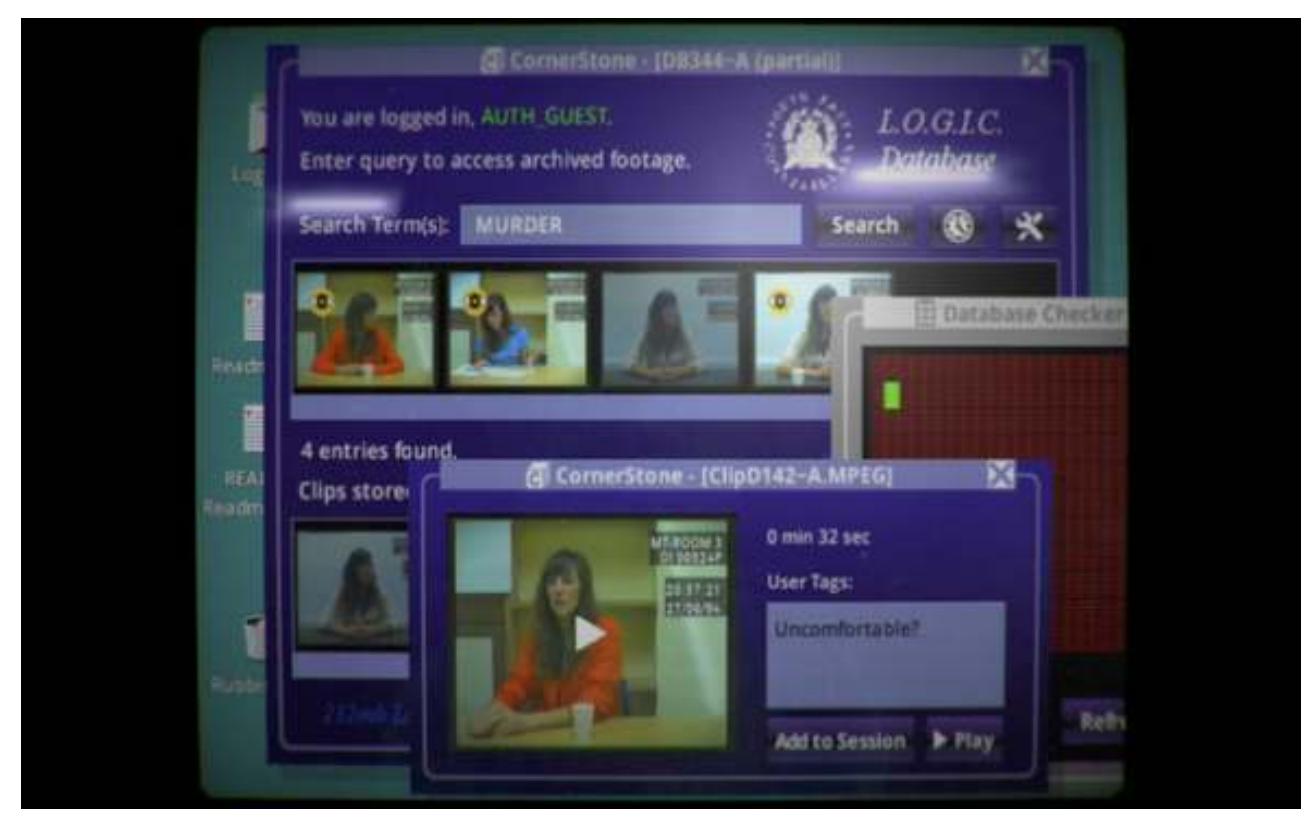

Figure 5 : Her Story (Sam Barlow 2015), interface de jeu et d'interrogation de la base de vidéos.

\subsection{Le langage comme mode d'action}

Le principe d'agir par l'intermédiaire du langage n'est pas exploité qu'en fiction interactive. Le jeu de Subservient Chicken ${ }^{14}$ (Crispin et al 2001), par exemple, ne fonctionne que par l'intermédiaire du langage (écrit ici), puisqu'il s'agit de donner des instructions à un coq géant par l'entremise du clavier. Là encore, une base de données de vidéos est activée et le clip correspondant à l'action demandée est joué.

16 Les robots conversationnels (par exemple Pandorabots ${ }^{15}$, A.L.I.C.E. AI Foundation 1995, figure 6) fonctionnent selon un principe similaire, sauf qu'il ne s'agit plus cette fois d'une base de données de clips vidéos, mais d'une base de données d'éléments de langage, le système reposant sur le traitement automatique du langage. 


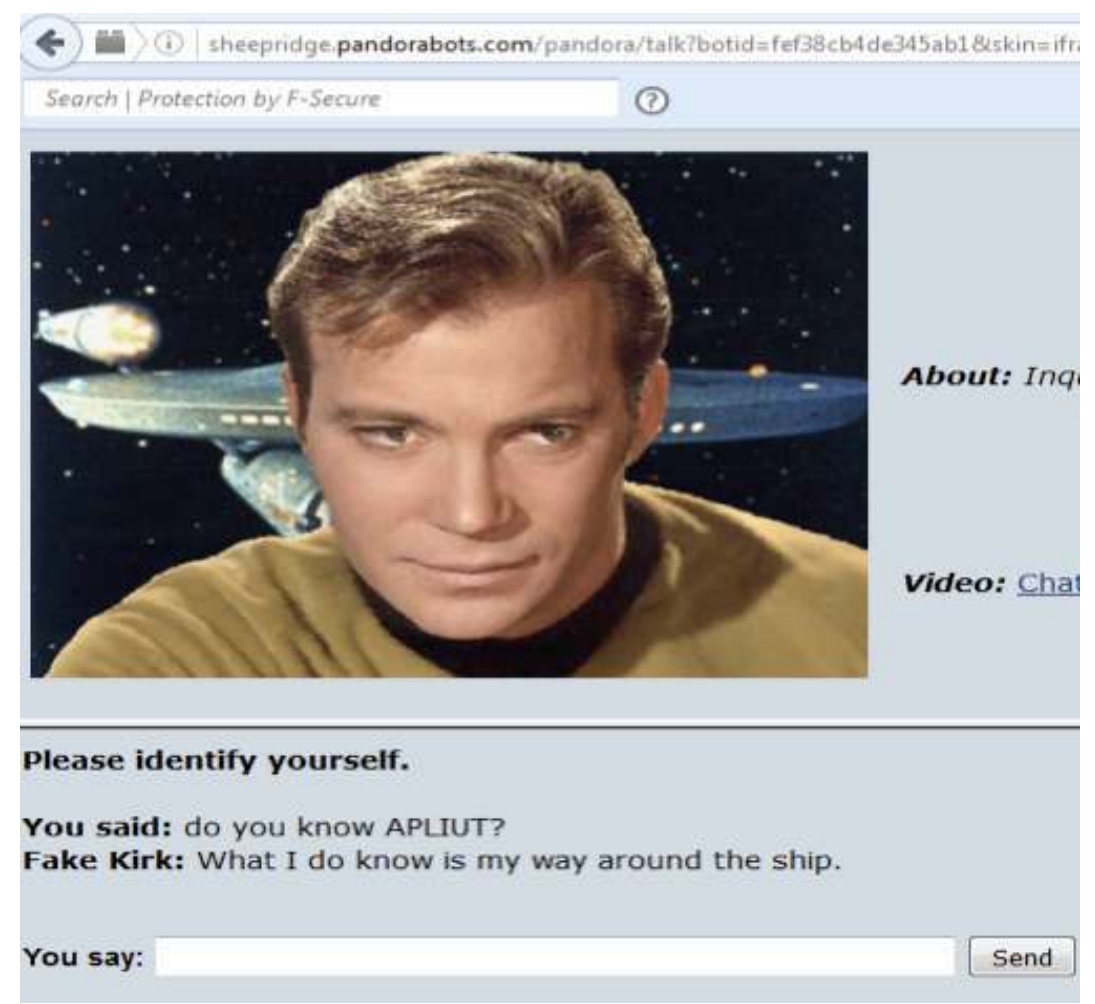

Figure 6 : Pandorabot, Fake Kirk (A.L.I.C.E. Al Foundation 1995) : robot conversationnel, interface de saisie de texte.

\section{Créer par le langage : l'étudiant démiurge}

17 «Le démiurge, c'est celui qui, s'assimilant à Dieu, prétend être littéralement 'l'auteur d'autrui' » (Meirieu 64). Dans le cas qui nous préoccupe, l'apprenant peut être auteur de sa propre ressource interactive à travers le langage tel un démiurge également (Rémon 347). En effet, si une manière d'utiliser le numérique pour la pratique de la langue est l'utilisation de ressources existantes clé en main, ou l'exploitation du principe de la combinatoire, il est aussi possible de devenir créateur de ressources ou d'univers virtuels.

Ainsi, dans un Moo (Multi-User Dimension Object-Oriented) (Tecfa Moo ${ }^{16}$, figure 7), le lieu virtuel existe par la description textuelle qui en est faite par l'utilisateur, éventuellement accompagnée d'images.

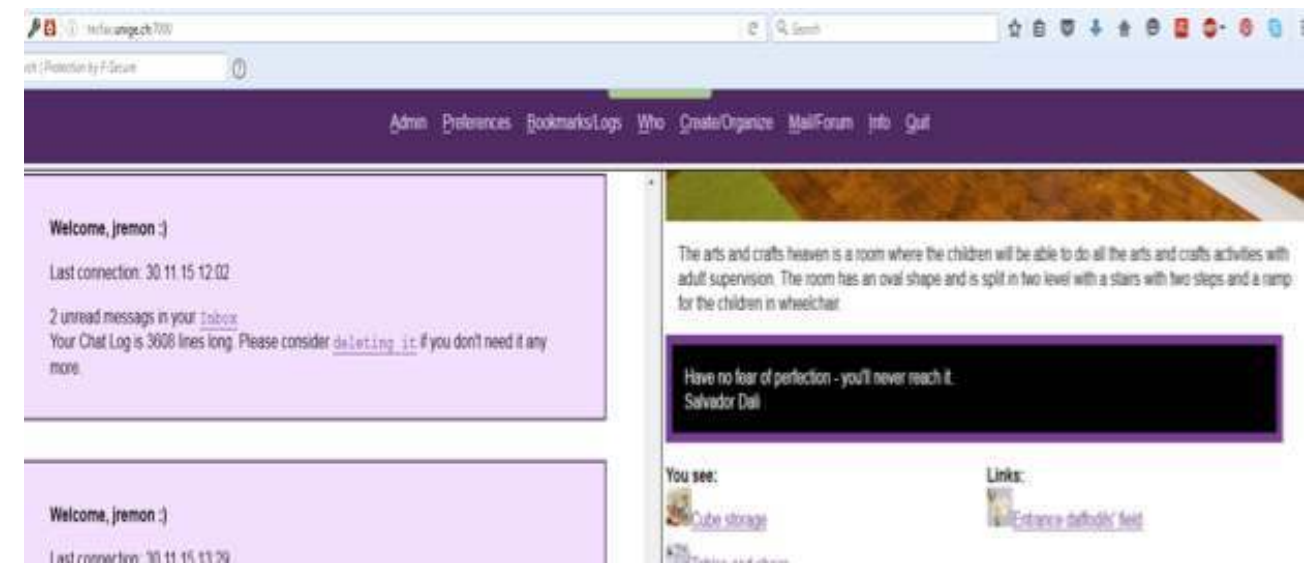

Figure 7 : Tecfa Moo : exemple de Moo, visualisation d'un espace virtuel, et de l'interface de création. 
Le jeu Wordseye ${ }^{17}$ (WordsEye Inc. 2016, figure 8) est également intéressant de ce point de vue. Une image est générée cette fois automatiquement à partir d'une description textuelle. Ainsi, dans l'exemple ci-dessous, la description textuelle est « The ambient light is navy. The camera light is navy. The sky is facing up. A shiny whale 30 feet above the ground, etc.

$\gg$

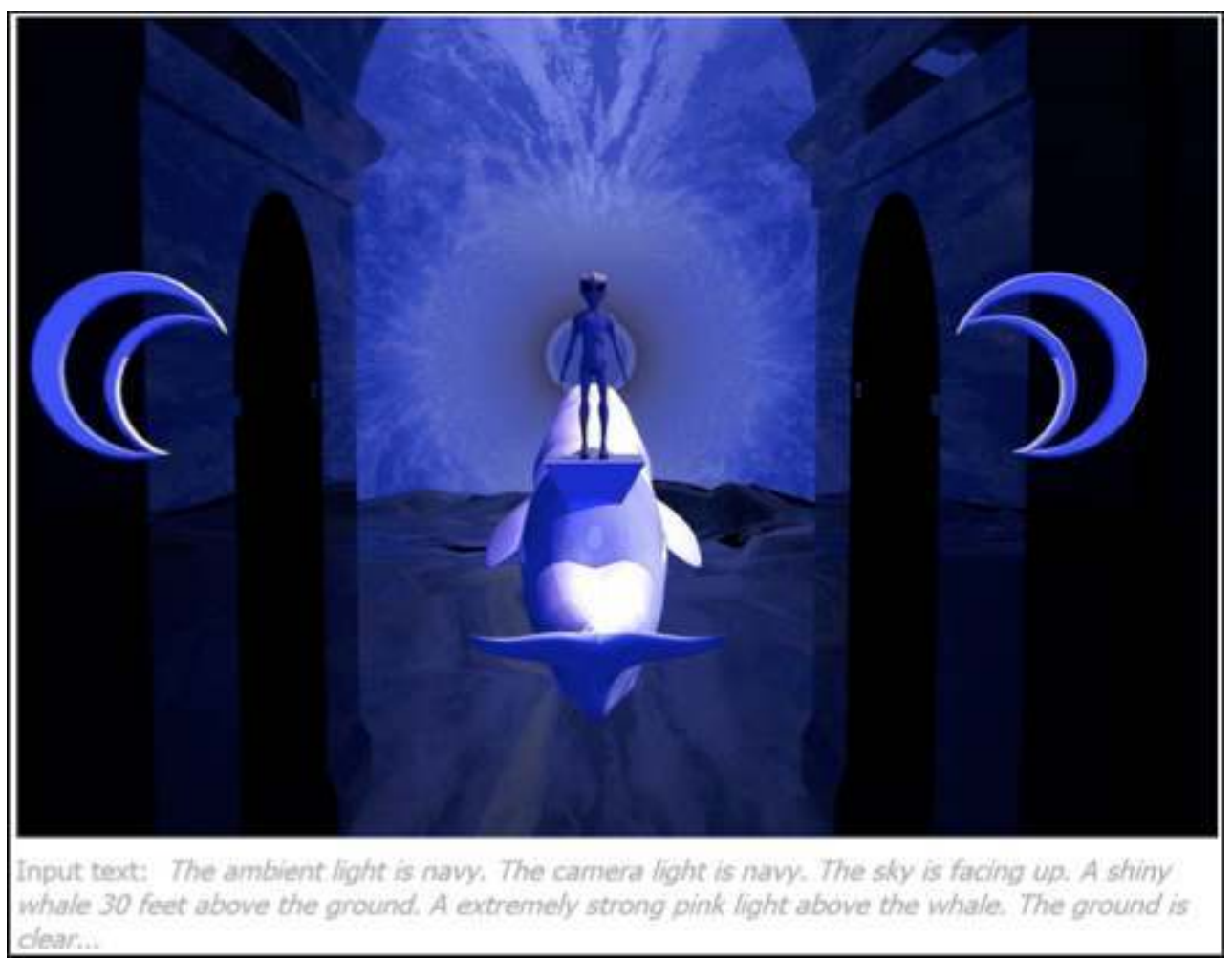

Figure 8 : Wordeye (WordsEye Inc. 2016) : exemple de création avec la description textuelle correspondante en-dessous.

20 Un principe similaire est utilisé avec l'application Imagequilts ${ }^{18}$, qui génère une mosaïque d'images issus de google image à partir de mots-clés, ou encore avec le site internet Wordle ${ }^{19}$ qui produit un nuage de mots à partir d'un texte.

21 Si dans les exemples ci-dessus on reste au stade de la manipulation de lexique, avec la fiction interactive, comme avec les robots conversationnels, on peut aussi passer du statut d'utilisateur à celui de créateur en produisant sa propre fiction interactive, cette fois une histoire complète à choix multiples tel que Adventure Cow ${ }^{20}$ (Adventure Cow 2015), ou son propre bot comme dans Playground ${ }^{21}$ (PandoraBots 2014, figure 9). 


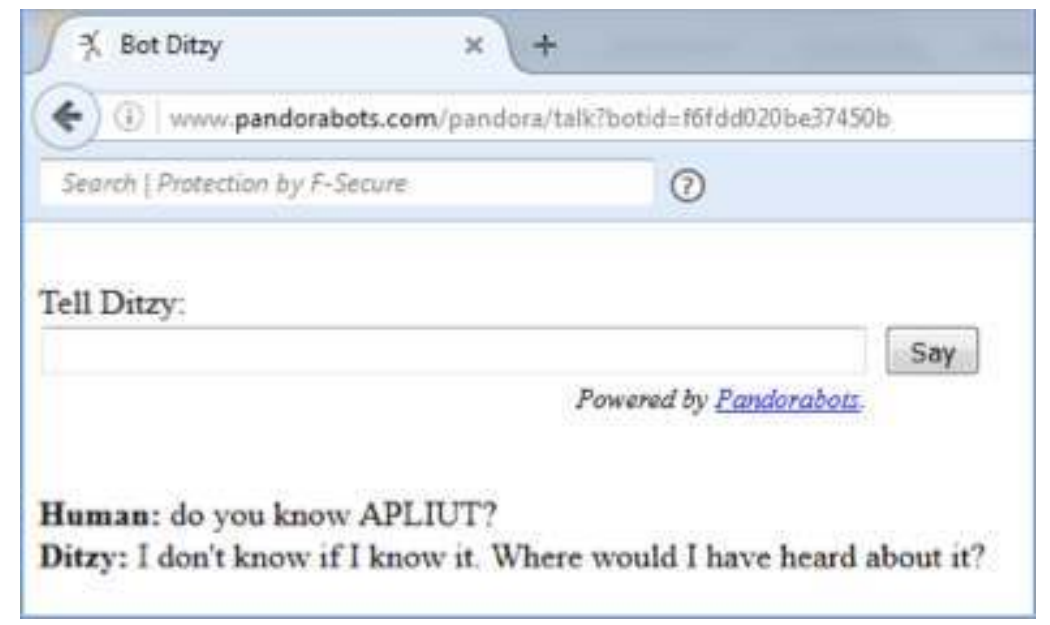

Figure 9 : Playground (PandoraBots 2014) : créer son propre bot conversationnel, interface d'interrogation du bot « Ditzy » créé par un étudiant.

\section{Jeu et spécialité}

Lors de l'exploitation de la plupart des ressources présentées ci-dessus, la spécialité étudiée peut être introduite, en plus de la langue étrangère. On peut par exemple facilement programmer le robot conversationnel pour répondre à des questions sur la spécialité, et de la même manière, on peut créer un scénario de fiction interactive à choix multiples qui fait référence au domaine de spécialité. On peut ainsi imaginer, pour des élèves ingénieurs nucléaires, de créer un scénario d'accident dans une centrale, avec les différents choix d'actions possibles à mener, décrits sous forme textuelle et hypertextuelle dans la langue cible.

D'autres ressources, des jeux sérieux notamment, s'ils peuvent être utilisés par des non spécialistes, peuvent aussi être exploités dans le cadre de la spécialité, comme la gestion ou l'environnement pour le jeu MacDonalds ${ }^{22}$ (McDonald's Videogame Molleindustria 2006).

Le jeu Stop disasters !23 (Playerthree and UN/ISDR 2007, figure 10) peut être utilisé avec des spécialistes de l'environnement ou de l'humanitaire, de même pour le documentaire interactif OffShore ${ }^{24}$ (Helios Design Labs 2013). 


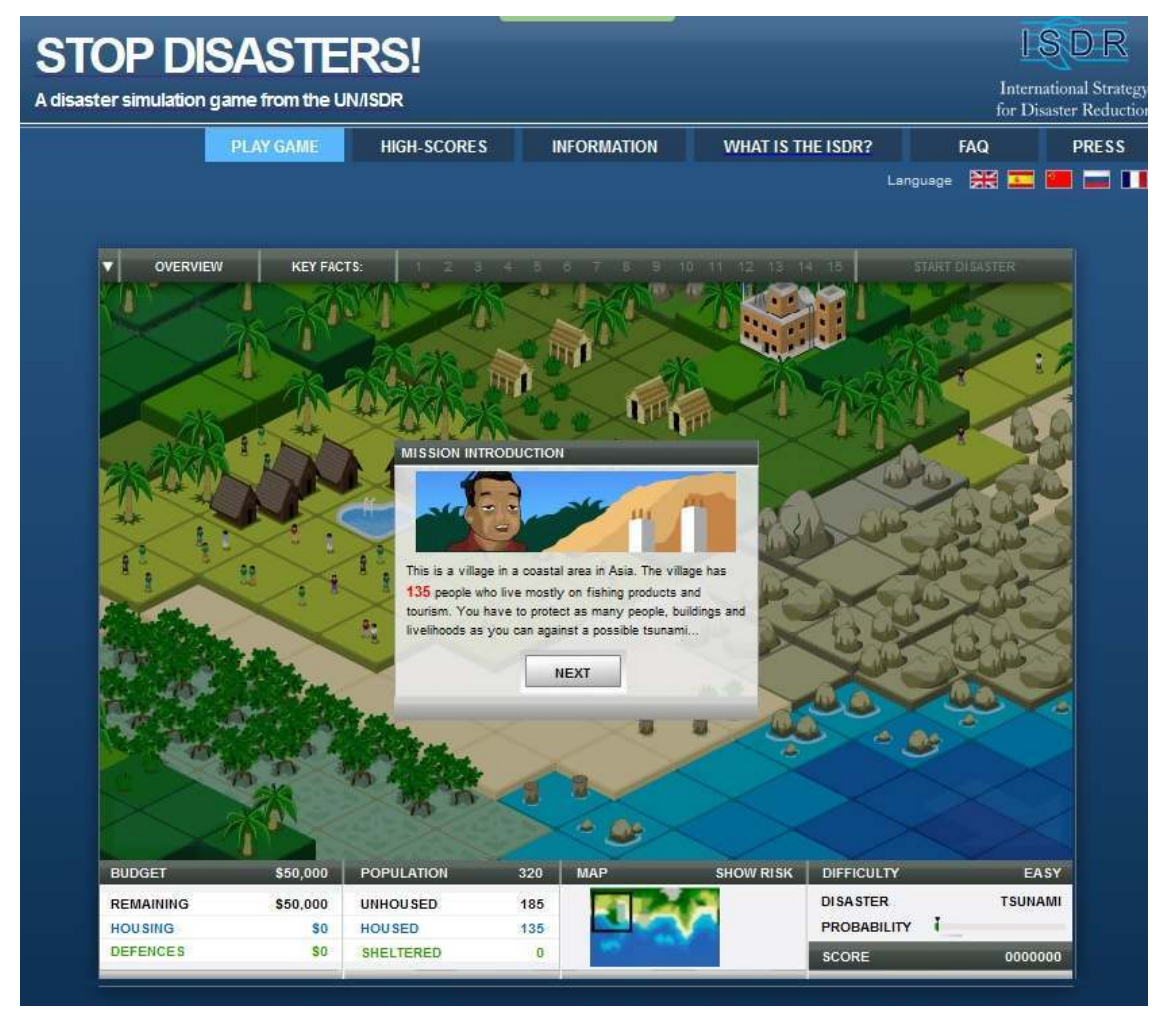

Figure 10 : Stop Disasters! (Playerthree and UN/ISDR, 2007), interface de jeu, introduction.

\section{De l'utilisation à la création}

Au terme de cette présentation rapide de ressources permettant de pratiquer la langue ou de créer par la langue, nous proposons une représentation à quatre niveaux de la relation de l'utilisateur à l'image (figure 11) et une seconde modélisation à quatre niveaux en fonction du rôle du langage dans l'accès au jeu (figure 12). Nous les commentons ci-après.

\subsection{De la fascination à l'interaction}

Nous avons évoqué la fascination exercée par l'écran en introduction. On ne niera pas un certain effet hypnotique de la lumière de l'écran, comme l'indique Frau-Meigs (24) :

un effet hypnotique, au sens où la lumière à l'écran transforme notre perception visuelle. La lumière, avec ses propriétés chromatiques et kinesthésiques, crée une mobilité qui entraîne une certaine immobilisation du regard de l'utilisateur, prisonnier de l'attente de ce qui va suivre, par captation. Celle-ci est renforcée par la présence de la lumière à l'intérieur du cadre.

Du point de vue de la luminosité et de l'image, nous distinguons dans la figure 11 la lumière de l'écran en tant que telle, c'est-à-dire celle que l'on voit même si on ne voit pas l'écran directement, d'un deuxième niveau dans lequel l'image fixe est présentée sur cet écran lumineux. À un troisième niveau, l'image est lumineuse et également animée, puis 
enfin, à un quatrième niveau, dans le cas de l'interactivité, l'image est lumineuse, animée et répond à nos actions.

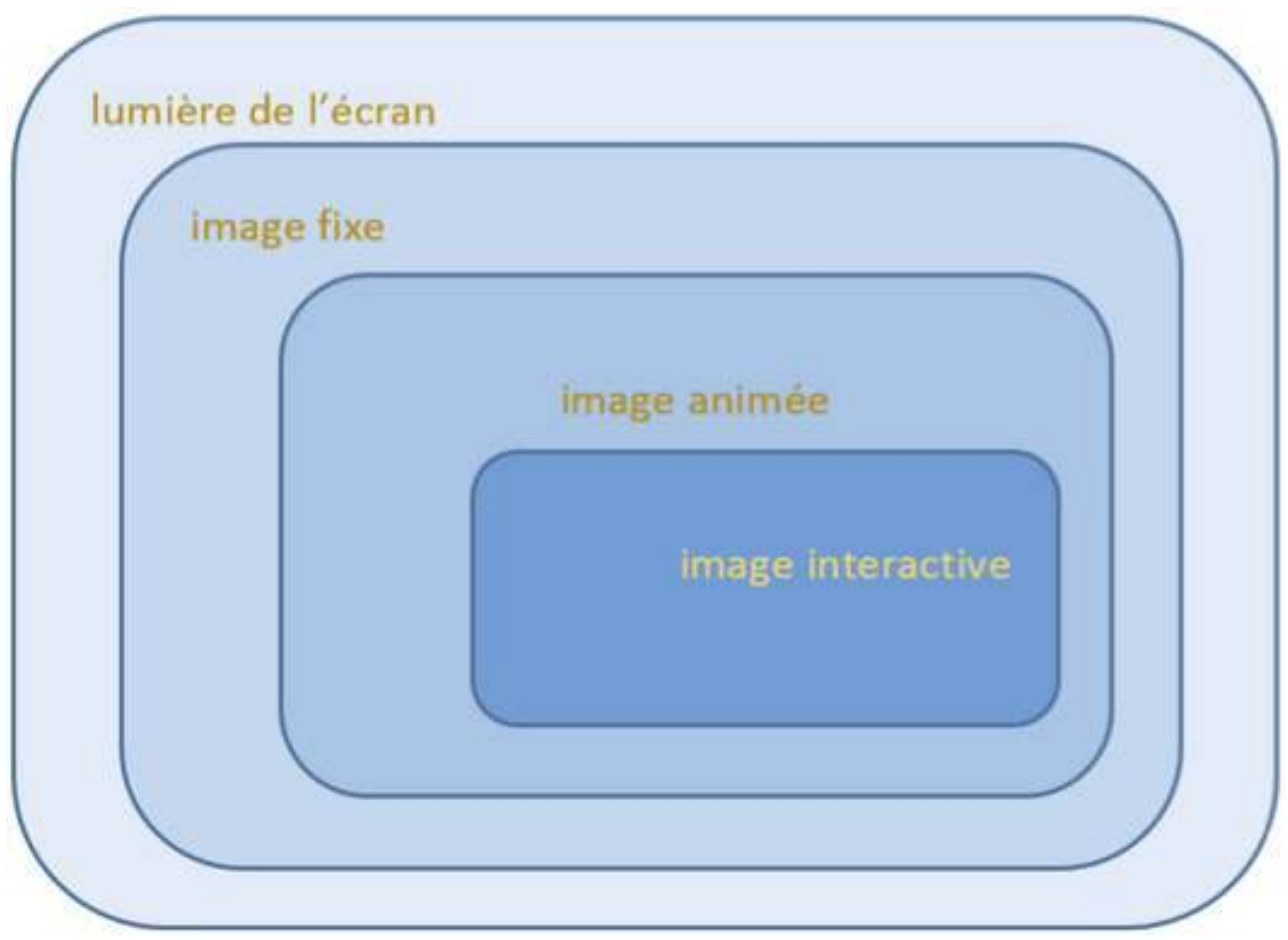

Figure 11 : représentation de la relation écran/lumière/image/interaction.

Ainsi que l'explique Soulage (49),

en abandonnant son destin fonctionnel et solitaire, l'écran informatique dès qu'il a été mis en réseau est devenu un écran global relié à une communauté médiatique réticulaire bousculant et détrônant l'agenda centralisateur et monolocutif $\mathrm{du}$ terminal-écran télévisuel.

\subsection{Langue/jeu/création}

En ce qui concerne l'image animée interactive, on peut tenter de représenter plusieurs niveaux, cette fois non plus autour de la relation écran/lumière/image/interaction, mais autour de la relation langue/jeu/création (figure 12). Le premier niveau est celui d'un jeu qui permet accessoirement une pratique de la langue (niveau 1). Ainsi, le jeu de création 3D Minecraft (Mojang 2011), au fort potentiel éducatif ${ }^{25}$ par ailleurs (collaboration, codage, transversalité), permet d'interagir éventuellement dans une langue étrangère mais il s'agirait plutôt dans ce cas d'un usage détourné de la ressource, dans laquelle les interactions langagières ne sont pas centrales. Le deuxième niveau est celui d'un jeu qui ne fonctionne que par l'intermédiaire du langage (niveau 2), comme nous en avons vu cidessus (Wordseye ou Subservient Chicken par exemple). Le troisième niveau est celui de la création même d'un jeu ou production numérique éventuellement ludique avec une implication du langage (niveau 3). Ainsi, on distingue : pratiquer la langue en jouant, jouer par le langage, et créer son propre jeu. On peut ajouter un dernier niveau au sein duquel, lors de la phase de création, on tient compte des caractéristiques propres à la discipline, par exemple en créant un robot conversationnel en anglais qui réponde à des questions juridiques simples (niveau 4). 


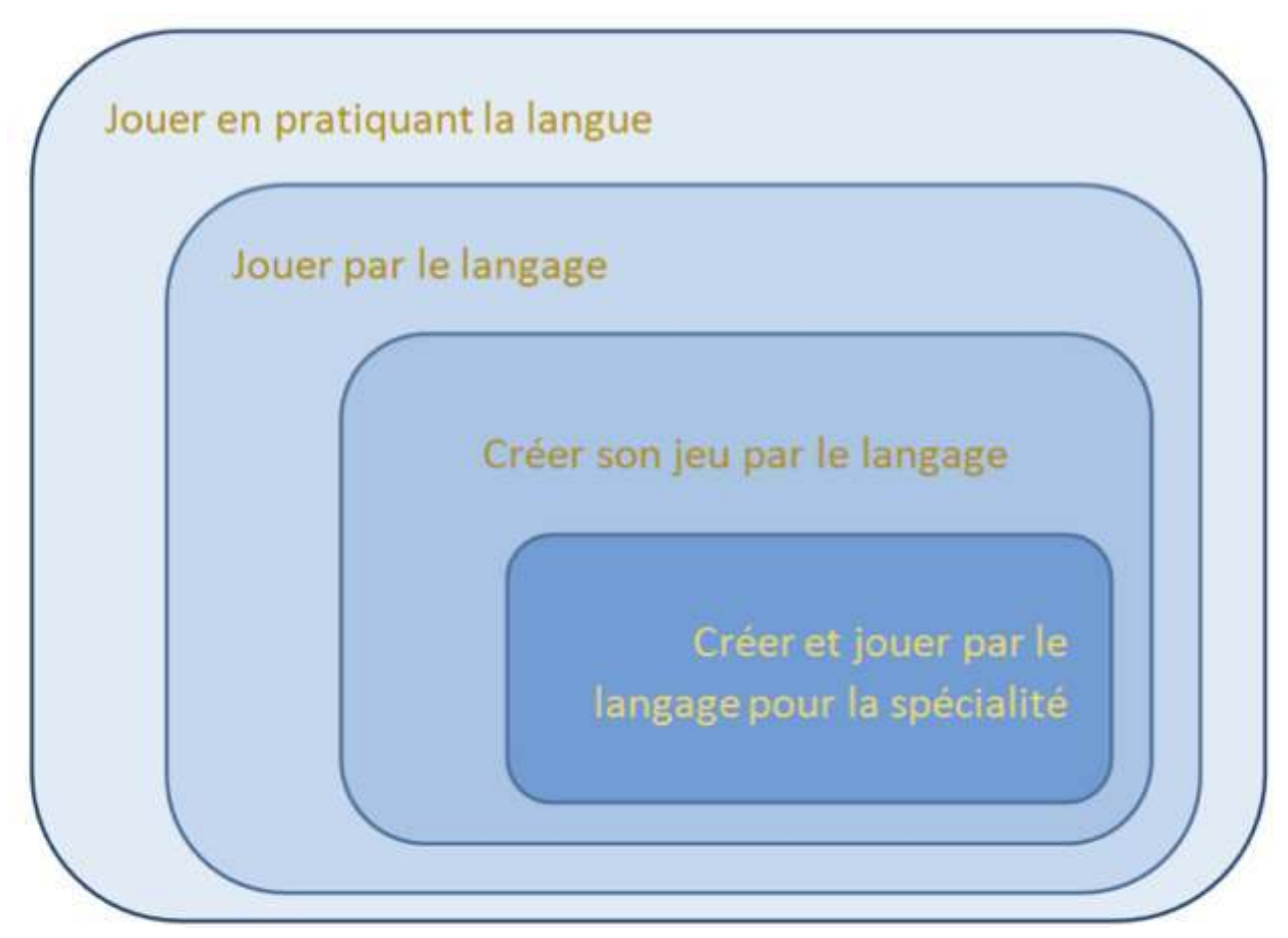

Figure 12 : Représentation de la relation entre langage, jeu et création.

\section{Conclusion : utiliser, pratiquer et créer}

Parmi les ressources que nous avons présentées ci-dessus (répertoriées, avec d'autres, sur le site PearlTrees $\left.{ }^{26}\right)$, certaines permettent de pratiquer la langue, d'autres non seulement permettent cette pratique, mais encore ne fonctionnent qu'à travers la langue. Sous forme de contrainte créatrice de langage, le jeu devient le langage lui-même, et le langage devient le jeu. Les ressources numériques, permettant d'aller de la pratique à la création en passant par l'interaction, offrent ainsi une variété d'applications que l'on pourra mettre à profit en fonction des profils d'apprenants, de la configuration de l'espace d'apprentissage et de l'intention pédagogique.

\section{BIBLIOGRAPHIE}

Frau-Meigs, Divina. « Technologies de la fascination. » Les cahiers de médiologie, vol. 2, n 10, 2000, pp. 178-187.

Chartier, Anne-Marie, et al. Lire, écrire. Tome 2 : produire des textes. Hatier Pédagogie, 1998.

Erwin, Ben, et al. « LEGO Engineer and RoboLab: Teaching Engineering with LabVIEW from Kindergarten to Graduate School. » International Journal of Engineering Education, vol. 16, $\mathrm{n}^{\circ}$ 3, 2000, pp. 181-192. 
Meirieu, Philippe. « Pédagogie et droits de l'homme. » Éduquer conformément aux droits de l'homme. Éditions Ouvrières, 1988, pp. 50-67.

Rémon, Joséphine. « MOO : l'apprenant de langue démiurge. » Apprentissages des langues et pratiques artistiques, dirigé par Joëlle Aden, Le Manuscrit, 2008, pp. 333-354.

Saussure, Ferdinand de. Cours de linguistique générale. Payot, 1994 (4éd. 1916).

Robinson, Hillary, et Sharrat Nick. Mixed-up fairy tales. Hodder, 2004.

Soulage, Pierre. « L'image écran, de la toile à l'interface. » MEI - Médiation et information, $\mathrm{n}^{\circ} 34$, 2012, pp. 43-52.

\section{NOTES}

1. amanita-design.net/samorost-1/

2. jayisgames.com/games/escaping-the-prison/

3. www.challengetheroom.fr/escape-game-grenoble/

4. www.buildwithchrome.com/builder

5. freerice.com/\#/english-vocabulary/1383

6. www.bbc.co.uk/bitesize/ks3/games/questionaut

7. www.britannica.com/quiz/browse

8. http://www.dckids.com/dc-super-friends/htmlgame/name/super-hero-me

9. http://www.makebeliefscomix.com/Comix/

10. https://www.fodey.com/generators/www.fodey.com/generators/

11. http://www.excitemike.com/twine/arsonsaurus.html

12. www.bbc.co.uk/programmes/articles/1g84m0sXpnNCv84GpN2PLZG/the-hitchhikers-guideto-the-galaxy-game-30th-anniversary-edition

13. www.herstorygame.com

14. oneweakness.com/subservient-chicken

15. alice.pandorabots.com/

16. tecfax.unige.ch:7000/

17. www.wordseye.com

18. www.imagequilts.com/

19. www.wordle.net

20. adventurecow.com

21. playground.pandorabots.com/en/

22. www.mcvideogame.com

23. www.stopdisastersgame.org/

24. offshore-interactive.com/

25. education.minecraft.net/

26. Ressources pour des jeux interactifs répertoriées sur le site PearlTrees à l'adresse suivante : http://www.pearltrees.com/c2i2e_anglais/interactive-games/id12542303 


\section{RÉSUMÉS}

Dans l'optique de redonner toute leur place aux jeux dans l'enseignement/apprentissage des langues, nous présentons des ressources numériques pour la pratique de l'anglais. Nous proposons des principes opérationnels et une classification empirique en fonction du rapport de ces ressources à la langue et à la créativité. Nous proposons aussi une réflexion sur la relation entre luminosité et interactivité. Si l'apprenant ou l'enseignant peuvent être utilisateurs de ressources, on peut aussi mettre en place des activités où la production langagière elle-même, et elle seule, permet la résolution de la tâche numérique.

In order to restore games to their rightful place in language teaching/learning, we present digital resources for the practice of English. We present operational principles and an empirical classification, according to the relation between these resources and both language and creativity. We also reflect upon the links between luminosity and interactivity. If the learner or the teacher can be users of these resources, one can also envisage activities where the language production itself, and nothing else, allows the resolution of the digital task.

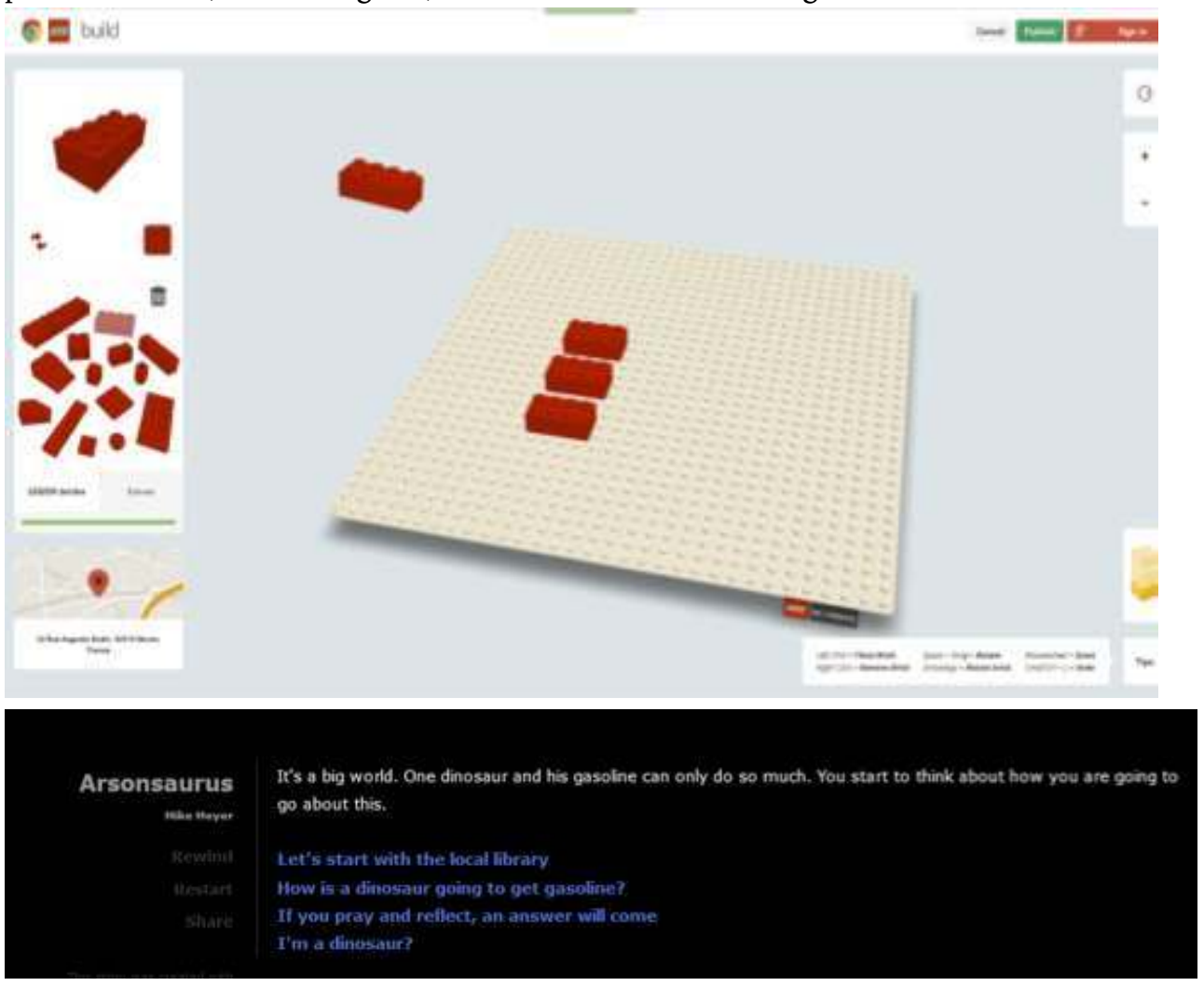




\section{INDEX}

Mots-clés : anglais, délégation d'écriture, jeux sérieux, TIC, interactivité

Keywords : English language, writing delegation, serious game(s), ICT, interactivity

\section{AUTEUR}

\section{JOSÉPHINE RÉMON}

Après des études en linguistique et informatique, Joséphine Rémon a été attachée linguistique à l'ambassade de France à Ottawa (Canada). Elle a consacré son doctorat à créer et implémenter des scénarios ludiques pour faire se rencontrer linguistique, apprentissage des langues et Internet. Elle enseigne l'anglais et les TIC à l'université Lyon 2, aux étudiants anglicistes, aux enseignants stagiaires, en Master Humanitaire et en Master Sciences de l'éducation à distance. Elle poursuit ses recherches au sein du laboratoire ICAR (UMR5191).Josephine.Remon@univ-lyon2.fr 